\title{
A RELEVÂNCIA DA COMPREENSÃO DO PROCESSO SAÚDE/DOENÇA POR ENFERMEIROS NA ATENÇÃO PRIMÁRIA
}

\section{THE RELEVANCE OF UNDERSTANDING OF THE HEALTH/DISEASE PROCESS BY NURSES IN PRIMARY CARE}

\author{
Renta Ferreira de Araújo ${ }^{1}$ \\ Anny Isabelly Medeiros de Goés ${ }^{2}$ \\ Francilene Maciel Ferreira da Silva ${ }^{3}$ \\ Karén Kelyany Duarte Costa ${ }^{4}$ \\ Laura Aires Cavalcante Leite ${ }^{5}$
}

RESUMO: A saúde coletiva é uma área essencial da atuação do enfermeiro e para que haja um atendimento qualificado, faz-se necessário a compreensão do processo saúde- doença pelos profissionais. OBJETIVO: Discutir o que a literatura cientifica da área traz a respeito da importância do entendimento dos enfermeiros da Atenção Primária de Saúde sobre o processo saúde-doença. MÉTODOS: Revisão integrativa, com busca na Biblioteca Virtual em Saúde que utilizou cruzamentos entre os descritores das ciências da saúde. Foram utilizados como critérios de inclusão: artigos em português, disponíveis na integra e de acesso aberto. Foram excluídas as produções repetidas. O material resultante da busca foi analisado com base na visão crítica dos autores que utilizaram um quadro sinóptico elaborado em Word para este fim. RESULTADOS: A seleção resultou em seis artigos que versam sobre: concepções e competências do enfermeiro na área de saúde coletiva, o trabalho como determinante do processo saúde-doença, processo saúde/doença e estratégia de saúde da família sob o olhar do usuário e conceitos e aplicabilidade da epidemiologia no Sistema Único de Saúde. CONCLUSÃO: Assim, o cuidado coletivo apresenta-se como peça chave no processo, delegando responsabilidades diferentes a todos os familiares e profissionais de saúde envolvidos.

\footnotetext{
1 Graduanda em Enfermagem pela Universidade Estadual da Paraíba e bolsista de Iniciação Científica CNPq, UEPB;

2 Graduanda em Enfermagem pela Universidade Estadual da Paraíba e bolsista de Iniciação Científica CNPq, UEPB;

${ }^{3}$ Graduanda em Enfermagem pela Universidade Estadual da Paraíba;

${ }^{4}$ Graduanda em Enfermagem pela Universidade Estadual da Paraíba;

${ }^{5}$ Graduanda em Enfermagem pela Universidade Estadual da Paraíba.
} 
Palavras chave: Atenção Primária à Saúde. Enfermagem em Saúde Comunitária. Processo Saúde-Doença.

ABSTRACT: Public health is an essential area of the nurse's work and in order for there to be qualified care, it is necessary for professionals to understand the healthdisease process. OBJECTIVE: To discuss what the scientific literature in the area brings about the importance of nurses' understanding of Primary Health Care about the health-disease process. METHOD: Integrative review, searching the Virtual Health Library that used intersections between health science descriptors. Inclusion criteria were: articles in Portuguese, available in full and open access. Repeated productions were excluded. The material resulting from the search was analyzed based on the critical view of the authors who used a synoptic table prepared in Word for this purpose. RESULTS: The selection resulted in six articles that deal with: nurses' conceptions and competencies in the collective health area, work as a determinant of the health- disease process, health / disease process and family health strategy under the user's view and concepts and applicability of epidemiology in the Unified Health System. CONCLUSION: Thus, collective care is presented as a key part in the process, delegating different responsibilities to all family members and health professionals involved.

Keywords: Primary Health Care. Community Health Nursing. Health-Disease Process. 


\section{INTRODUÇÃO}

A saúde coletiva é uma área essencial da atuação profissional do enfermeiro, na qual têm autonomia e segurança. No campo interdisciplinar, intersetorial e multiprofissional, de grande abrangência do SUS para atuar estrategicamente procura-se compreender o processo saúde-doença e seus determinantes e desenvolver ações visando à integralidade dos indivíduos atendidos, realizar educação em saúde nas diversas áreas temáticas que é cabível ao enfermeiro promoção de saúde e desenvolver pesquisas como também incentivar pesquisadores para área. Além disso, a sistematização da assistência da enfermagem para um cuidado efetivo trazendo melhorias para o indivíduo que necessita atendimento (REGIS, 2015).

Um dos pontos importantes para compreensão do processo saúde-doença, como: os fatores determinantes e condicionantes na própria aplicabilidade no âmbito do SUS.

Sendo compreensível os modelos de atenção de saúde no Brasil e a necessidade do conhecimento de cada um deles para uma integralidade do indivíduo. Os modelos: biomédico, sanitarista, modelo determinação- social da saúde, modelo ecológico e o modelo sistêmico, esses são alguns exemplos da infinidade de modelos e a diversidade na Atenção Primária à Saúde (REGIS, 2015).

É necessário compreender que não basta conhecer o funcionamento das doenças e encontrar mecanismos para seu controle, mas também, mecanismos de promoção da saúde diz respeito ao fortalecimento da saúde por meio da construção da capacidade de escolha; da mediação entre as pessoas e seu ambiente, combinando escolhas individuais com responsabilidade social pela saúde (MASCARENHAS; MELO; FAGUNDES, 2012).

A Atenção Primária à Saúde (APS), possui em sua dimensão abrangente, representa um espaço privilegiado para as ações de promoção da saúde no âmbito do sistema de serviços. Isso se dá principalmente porque a APS é essencial para a 
estruturação de sistemas de saúde, quando compreendida como uma estratégia que possibilita a intervenção sobre os determinantes sociais do processo saúde-doençacuidado, que são inerentes a atenção à saúde da população (MASCARENHAS; MELO; FAGUNDES, 2012).

A importância dos enfermeiros ao atuarem no campo da atenção primária e em consonância com os pressupostos da promoção da saúde, devem pautar a sua prática em uma concepção ampliada de saúde, considerando os determinantes sócio-ambientais do processo saúde-doença-cuidado. Estimulando e promovendo a participação política da comunidade; devem atuar de modo a extrapolar os limites dos serviços de saúde, com vistas a adotar ações intersetoriais e criar ambientes favoráveis à saúde; e devem se engajar na luta pela consolidação de políticas públicas saudáveis (MASCARENHAS; MELO; FAGUNDES, 2012).

A sociedade brasileira se modificou ao longo dos tempos, um dos efeitos é a transição demográfica brasileira, com o perfil de morbi-mortalidade, que é um indicador relativamente sensível das condições de vida e do modelo de desenvolvimento de uma população. Sendo assim, o objetivo desse estudo é compreender por meio da literatura, a importância do acompanhamento na APS por enfermeiros nas Unidades Básicas de Saúde do processo saúde- doença, justificando por possuírem vínculos com a comunidade para assim, compreender o processo de adoecimento da comunidade/individuo com os fatores determinantes e condicionantes da realidade inserida.

\section{MÉTODO}

Trata- se de uma pesquisa bibliográfica sobre os modelos do processo saúdedoença, articulando-se com os temas interligados de epidemiologia. Nesse estudo, buscou-se compreender as características principais dos modelos explicativos da ocorrência de doenças e agravos e os fatores determinantes e condicionantes do processo saúde-doença. Como também, todo o processo histórico relacionado a transição epidemiológica no Brasil e a análise do sistema de saúde atual interligando 
a atuação da Enfermagem nas vivências da realidade na Atenção Primária nas Unidades Básicas de Saúde que é a porta de entrada do indivíduo e do meio de convívio.

Critérios de Inclusão: artigos dos últimos 5 anos, disponível de forma gratuita, em idioma Português, nas plataformas Scielo, Google acadêmico.

Utilizou-se de materiais disponíveis pelo Ministério da Saúde, Plataforma UNASUS e o livro: "Epidemiologia: conceitos e aplicabilidade no Sistema Único de Saúde" para subsidio para discussão dos artigos encontrados na busca.

A RELEVÂNCIA DA COMPREENSÃO DO PROCESSO SAÚDE/DOENÇA POR
ENFERMEIROS NA ATENÇÃO PRIMÁRIA: uma revisão integrativa.
\begin{tabular}{|l|l|}
\hline OBJETIVO & $\begin{array}{l}\text { Avaliar o conhecimento científico produzido inerente } \\
\text { ao processo saúde-doença por enfermeiros na } \\
\text { Atenção Primária à Saúde. }\end{array}$ \\
\hline QUESTÃO NORTEADORA & $\begin{array}{l}\text { "Quais as evidências científicas inerentes ao processo } \\
\text { saúde-doença de e as estratégias de enfermeiros na } \\
\text { Atenção Primária à Saúde?" }\end{array}$ \\
\hline FONTE DOS DADOS & Scielo, Google acadêmico. \\
\hline DESCRITORES & $\begin{array}{l}\text { Português: Processo Saúde-Doença, Enfermagem } \\
\text { em Saúde Comunitária, Atenção Primária à Saúde. } \\
\text { Inglês: Health-Disease Process, Community Health } \\
\text { Nursing, Primary Health Care. } \\
\text { Decs em espanhol: Proceso Salud-Enfermedad, } \\
\text { Enfermeria em Salud Comunitaria, Atención Primaria } \\
\text { de Salud. }\end{array}$ \\
\hline
\end{tabular}




\begin{tabular}{|c|c|}
\hline EXPRESSÕES DE BUSCA & 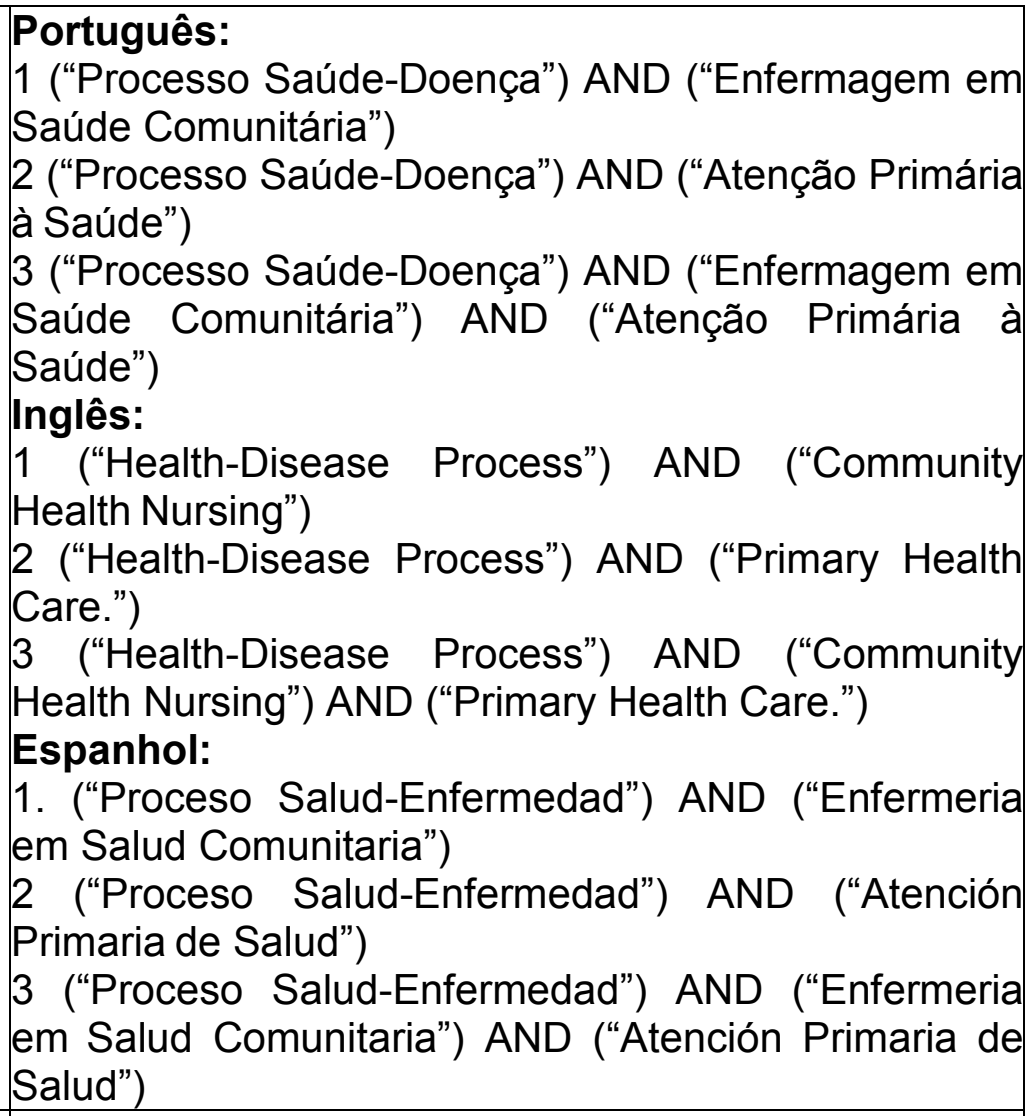 \\
\hline OPERADOR BOOLEANO & “"AND” \\
\hline CRITÉRIOS DE INCLUSÃO & $\begin{array}{l}\text { - Os estudos que respondem à questão } \\
\text { norteadora. } \\
\text { Artigos publicados nos últimos cinco anos, em } \\
\text { língua portuguesa ou espanhola; } \\
\text { Artigos disponíveis na íntegra e de forma } \\
\text { gratuita. }\end{array}$ \\
\hline CRITÉRIOS DE EXCLUSÃO & $\begin{array}{l}\text { - Quaisquer outros tipos de estudos que não } \\
\text { seja em formato de artigo científico; tais como artigos } \\
\text { em jornais, resumos de congresso, editoriais, teses, } \\
\text { dissertações enfim, toda a literatura cinzenta. }\end{array}$ \\
\hline $\begin{array}{l}\text { INSTRUMENTO DE COLETA } \\
\text { DE DADOS }\end{array}$ & URSI; GALVÃO, 2006 \\
\hline
\end{tabular}

\section{RESULTADOS}

Descrição dos artigos analisados no documento: 


\begin{tabular}{|c|c|c|c|c|c|}
\hline Identificacão & Autores & Periódico & Ano & $\frac{\text { Tipo de }}{\text { pesquisa }}$ & Principais resultados \\
\hline $\begin{array}{l}\text { Compreensão da } \\
\text { dinâmica familiar } \\
\text { no processo } \\
\text { saúde- doença e } \\
\text { intervenção pela } \\
\text { equipe de saúde } \\
\text { da família: um } \\
\text { estudo de caso. }\end{array}$ & 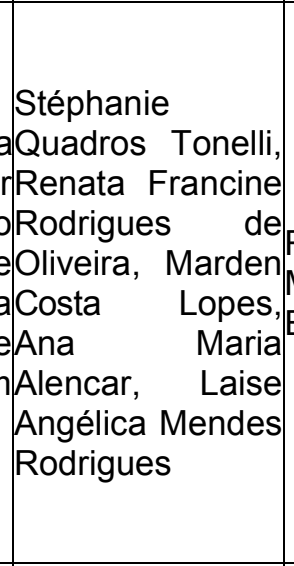 & $\begin{array}{l}\text { Revista Norte } \\
\text { Mineira de } \\
\text { Enfermagem }\end{array}$ & 2016 & $\begin{array}{l}\text { Estudo } \\
\text { Caso }\end{array}$ & $\begin{array}{l}\text { Analisa os aspectos essenciais } \\
\text { de um grupo familiar no } \\
\text { processo saúde doença, em um } \\
\text { diagnóstico de depressão. No } \\
\text { estudo, com utilização de } \\
\text { ferramentas de abordagem } \\
\text { efamiliar, e propor desfechos para } \\
\text { a família para o estabelecimento } \\
\text { de um cuidado de forma } \\
\text { dinâmica e coletiva. Além disso, } \\
\text { reconhecendo a importância do } \\
\text { vinculo das equipes de Saúde da } \\
\text { Família para um intervenção } \\
\text { efetiva no cuidado. }\end{array}$ \\
\hline $\begin{array}{l}\text { O enfermeiro na } \\
\text { área da saúde } \\
\text { coletiva: } \\
\text { concepções } \\
\text { competências }\end{array}$ & $\begin{array}{l}\text { Cristiano Gil } \\
\text { Regis, Nildo } \\
\text { Alves Batistal }\end{array}$ & $\begin{array}{l}\text { Revista } \\
\text { Brasileira de } \\
\text { Enfermagem }\end{array}$ & 2015 & $\begin{array}{l}\text { Qualitativa } \\
\text { de caráte } \\
\text { exploratório } \\
\text { descritivo }\end{array}$ & $\begin{array}{l}\text { Nesse artigo, apresenta uma } \\
\text { pesquisa qualitativa sobre o } \\
\text { ensino de saúde coletiva nos } \\
\text { cursos de graduação em } \\
\text { enfermagem das universidades } \\
\text { públicas da Região Norte do } \\
\text { Brasil. Reconhecendo a } \\
\text { importância da área de } \\
\text { er conhecimento e como acontece } \\
\text {-- há aplicação na prática } \\
\text { profissional. Compreendendo } \\
\text { que a saúde coletiva é uma área } \\
\text { essencial da formação } \\
\text { profissional da enfermagem e } \\
\text { constitui um campo importante } \\
\text { da atuação de enfermeiros e tem } \\
\text { o SUS como sua principal área } \\
\text { de estudo. }\end{array}$ \\
\hline $\begin{array}{l}\text { O trabalho como } \\
\text { determinante do } \\
\text { processo saúde- } \\
\text { doença }\end{array}$ & Ana Claudia & $\begin{array}{l}\text { Tempo } \\
\text { Social, } \\
\text { Revista de } \\
\text { Sociologia da } \\
\text { USP, v. 27, n. } \\
1\end{array}$ & 2015 & $\begin{array}{l}\text { Estudo } \\
\text { Caso }\end{array}$ & $\begin{array}{l}\text { No estudo busca compreender o } \\
\text { processo saúde-doença e a } \\
\text { relação do trabalho como } \\
\text { determinante. Se trata de um } \\
\text { relato de experiência, em que se } \\
\text { busca ao recorrer do relato sobre } \\
\text { seu processo de adoecimento no } \\
\text { trabalho parte do pressuposto de } \\
\text { que, para compreender a relação } \\
\text { entre trabalho e saúde, é } \\
\text { necessário basear-se na } \\
\text { vivência do sujeito. }\end{array}$ \\
\hline
\end{tabular}




\begin{tabular}{|c|c|c|c|c|c|}
\hline $\begin{array}{l}\text { Prática reflexiva } \\
\text { e formação } \\
\text { profissional: } \\
\text { aproximações } \\
\text { teóricas r } \\
\text { campo da Saúde } \\
\text { e }\end{array}$ & $\begin{array}{l}\text { Luciana } \\
\text { Kênia Lara } \\
\text { Marilia } \\
\text { Santos }\end{array}$ & $\begin{array}{l}\text { Escola } \\
\text { Nery }\end{array}$ & 2018 & & \begin{tabular}{|l} 
Trata-se de um pensamento \\
reflexivo de um currículo \\
inovador como expressão da \\
insatisfação ao paradigma da \\
fragmentação, visto que, no \\
campo da promoção da saúde, é \\
necessário mobilizar r a \\
reflexividade para ações e \\
contextos se baseando nos \\
escritos de Donald Schön e se \\
aplicam à prática do enfermeiro \\
para lidar com os condicionantes \\
e determinantes do processo \\
saúde-doença.
\end{tabular} \\
\hline $\begin{array}{l}\text { Subjetividade } \\
\text { saúde coletiva: } \\
\text { produção } \text { de } \\
\text { discursos na re-c } \\
\text { significação do } \\
\text { processo saúde- } \\
\text { doença no pós- } \\
\text { moderno }\end{array}$ & Cristian Fabian & $\begin{array}{l}\text { Revista } \\
\text { Subjetividade } \\
\text { s }\end{array}$ & 2016 & $\begin{array}{l}\text { Estudo } \\
\text { descritivo }\end{array}$ & $\begin{array}{l}\text { Este artigo se compreende na } \\
\text { construção da saúde a partir do } \\
\text { nascimento da medicina social, e } \\
\text { analisa o desenvolvimento do } \\
\text { conceito de saúde, } \\
\text { demonstrando as práticas em } \\
\text { saúde, o trabalho dos } \\
\text { profissionais neste campo e a } \\
\text { (des)organização do mundo } \\
\text { pósmoderno inferem novos } \\
\text { significados para o processo de } \\
\text { saúde-doença ou para a } \\
\text { representação da enfermidade. }\end{array}$ \\
\hline $\begin{array}{l}\text { Processo } \\
\text { saúde/doença e } \\
\text { estratégia de } \\
\text { saúde da família: } \\
\text { o olhar do } \\
\text { Usuário. }\end{array}$ & 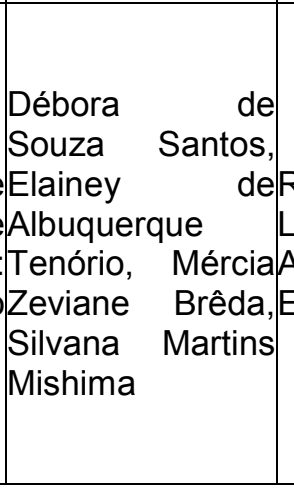 & $\begin{array}{l}\text { Revista } \\
\text { Latino- } \\
\text { Americana de } \\
\text { Enfermagem }\end{array}$ & 2014 & $\begin{array}{l}\text { Estudo } \\
\text { qualitativo }\end{array}$ & $\begin{array}{l}\text { Neste artigo, analisa os } \\
\text { significados atribuídos pelos } \\
\text { usuários da Atenção Primária à } \\
\text { Saúde ao seu processo de } \\
\text { saúde/doença e aos serviços } \\
\text { utilizados, são dois grupos } \\
\text { distintos (idosos/gestante) na } \\
\text { compreensão das ralas, } \\
\text { característica do do estudo } \\
\text { qualitativo para a importância da } \\
\text { atenção primaria sobre o olhar } \\
\text { do usuário do serviço. }\end{array}$ \\
\hline
\end{tabular}

Diante dessa temática, é necessário o fortalecimento dos espaços dialógicos já existentes e o fomento para a criação de novos, onde usuários e profissionais de saúde da ESF possam expressar suas percepções, sentimentos e desejos relativos às práticas de saúde, considerando- se a vivência subjetiva de seu processo de saúde/doença nas ações de saúde, revelando a autonomia do usuário para o autocuidado na promoção da saúde (SANTOS, 2014).

Assim, deve-se acontecer a valorização das subjetividades dos usuários pelos profissionais de saúde, com ações de educação em saúde e articulação de apoio intersetorial para a população, contribui para reversão do modelo de atenção nessa 
realidade, ao potencializar a produção de práticas de saúde comprometidas com a promoção da saúde, integralidade e participação (SANTOS, 2014).

\section{CONCLUSÃO}

Tonelli (2016) ressalta a importância do grupo familiar no processo saúde doença, transformando, assim, a família em um potencial de saúde do indivíduo. Além disso, o cuidado coletivo apresenta-se como peça chave no processo, delegando responsabilidades diferentes a todos os familiares e profissionais de saúde envolvidos. Para isso, torna-se necessário o vínculo das Equipes de Saúde da Família com o contexto único de cada paciente usuário do serviço.

Além disso, torna-se evidente a importância da capacitação dos profissionais de saúde quanto ao contexto da saúde coletiva desde a graduação. Segundo Regis (2015), a saúde coletiva é uma área extremamente explorada pelo enfermeiro, fato que torna cada vez mais necessário a aproximação do estudante de enfermagem com a realidade enfrentada pela mesma. Além disso, um dos principais objetivos de estudo da saúde coletiva é o Sistema Único de Saúde, campo de atuação de grande maioria dos enfermeiros.

Com todo esse contexto, com pontos positivos e negativos, podemos afirmar que a enfermagem encontra-se no elo entro o indivíduo, comunidade e serviço, sendo essa relação facilitadora no processo saúde/doença, porém essa relação entre o usuário e os profissionais de saúde devem estreitar cada vez mais laços para facilitar o cuidado dos sujeitos na APS.

A relação entre usuário e serviço limita alguma das partes as relações não têm o devido espaço no cotidiano do serviço, as ações em saúde tendem a ser padronizadas, verticalizadas e excludentes, ou seja, desumanizam o serviço sendo cada vez mais nominativo para o SUS características. Deve-se estabelecer um encontro com os princípios da humanização em saúde, da autonomia e corresponsabilização dos atores que constroem os serviços de saúde.

Por isso, é necessário na relação entre serviço e usuário, há necessidade de oportunizar e potencializar espaços democráticos de decisão, que valorizem os 
significados dos sujeitos implicados no processo de produção da saúde, para estabelecimento de vínculos que viabilizem a reversão do modelo de atenção no contexto da APS.

\section{REFERÊNCIAS BIBLIOGRÁFICAS}

ANDRADE, Luiz Odorico Monteiro de; BARRETO, Ivana Cristina de Holanda Cunha; BEZERRA, Roberto Cláudio. Atenção primária à saúde e estratégia saúde da família. In: Tratado de Saúde Coletiva, p. 783-835, 2006.

CAMARGO, Angela Maria et al. Abordagens grupais em saúde coletiva: a visão de usuários e de profissionais de enfermagem. Revista de Atenção à Saúde, v. 10, n. 31, 2012.

CARVALHO, Carolina Abreu de; PINHO, Judith Rafaelle Oliveira; GARCIA, Paola Trindade. Epidemiologia: conceitos e aplicabilidade no Sistema Único de Saúde. 2017. (LIVRO).

CARDOSO, Ana Claudia Moreira. O trabalho como determinante do processo saúde-doença. Tempo Social, v. 27, n. 1, p. 73-93, 2015.

FALKENBERG, Mirian Benites et al. Educação em saúde e educação na saúde: conceitos e implicações para a saúde coletiva. Ciência \& Saúde Coletiva, v. 19, p. 847-852, 2014.

GOMES, Karine de Oliveira et al. Atenção Primária à Saúde-a" menina dos olhos" do SUS: sobre as representações sociais dos protagonistas do Sistema Único de Saúde. Ciência \& Saúde Coletiva, v. 16, p. 881-892, 2011.

GUIMARÃES, Cristian Fabiano; MENEGHEL, Stela Nazareth. Subjetividade e saúde coletiva: produção de discursos na re-significação do processo saúde-doença no pós- moderno. Revista Subjetividades, v. 3, n. 2, p. 353-371, 2016.

MASCARENHAS, Nildo Batista; MELO, Cristina Maria Meira; FAGUNDES, Norma Carapiá. Produção do conhecimento sobre promoção da saúde e prática da enfermeira na Atenção Primária. Revista Brasileira de Enfermagem, v. 65, n. 6, p. 991-999, 2012.

OLIVEIRA, Dora Lúcia Liedens Corrêa. A enfermagem e suas apostas no autocuidado: investimentos emancipatórios ou práticas de sujeição?. Revista Brasileira de Enfermagem, v. 64, n. 1, p. 185-188, 2011.

REGIS, Cristiano Gil; BATISTA, Nildo Alves. O enfermeiro na área da saúde coletiva: concepções e competências. Revista Brasileira de Enfermagem, v. 68, n. 5, p. 830-836, 2015.

SANTOS, Débora de Souza et al. Processo saúde/doença e estratégia de saúde da família: o olhar do usuário. Revista Latino-Americana de Enfermagem, v. 22, n. 6, p. 918-925, 2014.

TONELLI, Stéphanie Quadros et al. Compreensão da dinâmica familiar no processo saúdedoença e intervenção pela equipe de saúde da família: um estudo de caso. Renome, v. 5, n. 1, p. 74-84, 2016.

MASCARENHAS, Nildo Batista; MELO, Cristina Maria Meira; FAGUNDES, Norma Carapiá. Produção do conhecimento sobre promoção da saúde e prática da enfermeira na Atenção Primária. Revista Brasileira de Enfermagem, v. 65, n. 6, p. 991-999, 2012. 